\title{
Towards a Third Generation of Control Systems
}

\author{
P.R. Kumar \\ Department of Electrical and Computer Engineering, \\ University of Illinois, Urbana-Champaign
}

\begin{abstract}
The first generation of control systems can be regarded as analog control and the second generation as digital control. Over the past three decades since the advent of digital control, there have been great technological advances in computing hardware and software as well as in networking. We are therefore at the cusp of a third generation of control systems which consist of sensors and actuators connected by shared wired or wireless networks, and involving powerful computational nodes as well as software services.

How does one facilitate the proliferation of such next generation control systems? We argue that it is important to develop the appropriate abstractions and a matching architecture for the (re)convergence of control with communication and computation. We propose an abstraction of virtual collocation to be manufactured by the supporting middleware, and a principle of local temporal autonomy for enhancing reliability. We provide an overview of efforts in the Convergence Laboratory at the University of Illinois.
\end{abstract}

